\title{
Smart Energy Systems for Increasing the Energy Independence of Small Islands: Integrating Renewable Energies, Storage Systems and Sustainable Mobility
}

\author{
Davide Astiaso Garcia \\ Department of Astronautical, Electrical and Energy Engineering - DIAEE \\ Sapienza University of Rome \\ davide.astiasogarcia@uniroma1.it
}

Several islands around the world are facing common challenges in terms of high energy costs, local CO2 emissions, security of supply and system stability. In EU, many islands have become sites of energy innovation, where betting on Renewable Energy Sources (RES) is a winning choice to meet their energy needs. In this framework, renewable energies play a key role for supporting the transition of small islands to an autonomous, cleaner and low-carbon energy system - in line with the overall EU Energy Union package and EU2030 goals. The intervention will deal with the suitability of smart energy systems in the insular context. Particularly, the use of several RES generators in the grid will be discussed as well as the potential synergies that can be obtained by coupling different energy sectors such as the sectors of transport, heating and water production. In detail, the benefits in terms of enhanced grid flexibility and thus the potential increase of RES penetration without compromising the island electric grid will be presented. Particularly, the speech will consider solutions that exploit synergies between different energy consuming sectors trough power-to-X solutions. Where $\mathrm{X}$ stands for i) transport through the use of Electric Vehicles (EVs), ii) heating by means of Heat Pumps (HPs) and iii) hydrogen produced by electrolysis used in Fuel-cell Electric Vehicles (FCEVs) or in Hydrogen Compressed and Natural Gas (HCNG) blend fuelled buses in the public transportation sector. All of the above-mentioned solutions will be presented by means of several energy scenarios applied to the island of Favignana, Italy. Results will be presented and discussed focusing on the energetic point of view as well as the economic and the environmental aspects. The intervention will end proposing interesting hint for future research topics related to innovative market schemes able to trigger the above-mentioned solutions and also the cascade effect that RES investments have on island communities. 